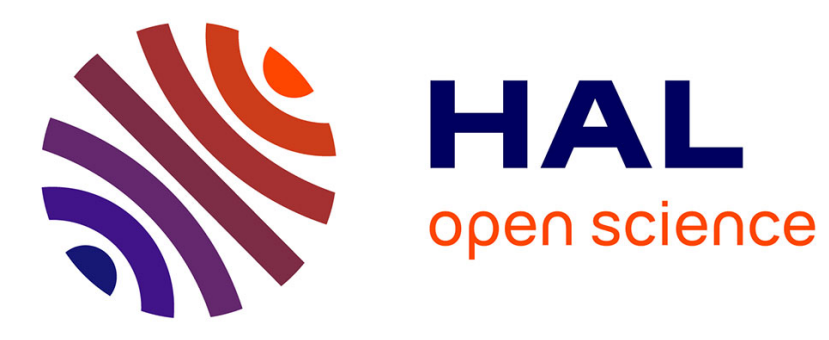

\title{
Les besoins de recherche en agroforesterie
}

Daniel Auclair, Francis Cailliez

\section{To cite this version:}

Daniel Auclair, Francis Cailliez. Les besoins de recherche en agroforesterie. Revue forestière française, 1994, 46 (S), pp.141-151. 10.4267/2042/26610 . hal-03444338

\section{HAL Id: hal-03444338 \\ https://hal.science/hal-03444338}

Submitted on 23 Nov 2021

HAL is a multi-disciplinary open access archive for the deposit and dissemination of scientific research documents, whether they are published or not. The documents may come from teaching and research institutions in France or abroad, or from public or private research centers.
L'archive ouverte pluridisciplinaire HAL, est destinée au dépôt et à la diffusion de documents scientifiques de niveau recherche, publiés ou non, émanant des établissements d'enseignement et de recherche français ou étrangers, des laboratoires publics ou privés. 


\title{
LES BESOINS DE RECHERCHE EN AGROFORESTERIE
}

\author{
D. AUCLAIR - F. CAILLIEZ
}

Les articles précédents ont présenté divers aspects de l'agroforesterie, telle qu'elle est pratiquée en France ou ailleurs dans le monde, soit sous forme traditionnelle depuis des siècles, soit sous des formes modernisées. En étudiant ces systèmes, on constate cependant de nombreuses lacunes dans les connaissances, provenant en grande partie de la séparation entre les disciplines scientifiques concernées. En effet, le développement des sciences agronomiques (au sens large, incluant la foresterie) a favorisé cette séparation, pour deux raisons principales (Auclair, 1993) :

- D'une part, les progrès de l'agriculture moderne ont entraîné le développement de monocultures intensives, avec une nette séparation des différentes productions dans l'espace, les systèmes agroforestiers étant tombés en désuétude et jugés peu productifs.

- D'autre part, les progrès de la recherche, fondamentale et appliquée, ont favorisé le développement de disciplines permettant d'approfondir les connaissances sur le fonctionnement de chacune des parties, mais les sciences de synthèse ont progressé moins rapidement.

Si les systèmes basés sur la monoculture plus ou moins intensive ont encore largement leur place en France et dans le monde - certes, avec des adaptations visant à réduire les intrants et les coûts de production et à améliorer les effets sur l'environnement -, une meilleure intégration des activités agricoles et forestières est hautement souhaitable dans de nombreuses situations. Le développement des sciences de synthèse, nécessaire dans le contexte scientifique général, devient indispensable en ce qui concerne l'utilisation du territoire. Les questions posées par l'agroforesterie imposent une approche pluridisciplinaire, car s'y mêlent étroitement des objets biologiques et techniques à d'autres relevant des sciences sociales. En effet, le champ est vaste puisqu'il concerne aussi bien la sélection d'essences adaptées ou l'écophysiologie de cultures associées que la conduite d'arbres à faible espacement ou d'associations pluristratifiées, ainsi que l'organisation du travail dans des exploitations en cours de diversification, la gestion et la récolte des bois des exploitations agricoles, le marché du bois, l'attitude des agriculteurs et des propriétaires fonciers vis-à-vis de nouveaux enjeux et l'écologie du paysage... pour ne citer que quelques exemples. Cette problématique peut en outre s'élargir à d'autres questions comme celles relatives à la qualité des eaux et au rôle de filtre-puits que peuvent jouer les arbres vis-à-vis des polluants minéraux, organiques et xénobiotiques.

Des recherches sur l'agroforesterie se poursuivent depuis déjà une vingtaine d'années, mais principalement dans les pays du Sud où de nombreuses pratiques traditionnelles impliquent une gestion conjointe de productions agricoles et d'arbres, et en particulier avec le développement de I'ICRAF (Centre international pour la Recherche en Agroforesterie, au Kenya), de I'ICRISAT (Institut international de Recherche agronomique pour les Tropiques semi-arides, en Inde) et du CATIE (Centre de Recherche et d'Enseignement agronomique, Costa-Rica) (Jarvis, 1991). Un récent symposium international sur le thème: "Agroforesterie et utilisation du territoire dans les pays industrialisés" (Berlin, 30 mai au 2 juin 1994) a mis en évidence le faible développement des recherches en agroforesterie dans les pays tempérés. Elles se sont développées principalement en Nouvelle-Zélande (Dupraz et al., 1992 ; Knowles, 1991) et, dans une moindre mesure, aux ÉtatsUnis (Gold et Hanover, 1987 ; McDicken et Vergara, 1990) et en Chine (Zhu et al., 1991). L'IUFRO 


\section{AUCLAIR - F. CAILLIEZ}

(Union internationale des Instituts de Recherche forestière) s'intéresse à ce sujet avec le "groupe opérationnel" P1.15-00, actuellement en cours de réorganisation (création de quatre groupes de travail sur agroforesterie tropicale, agroforesterie tempérée, recherche fondamentale et modélisation, sciences sociales).

En Europe, des opérations de recherche se mettent en place grâce à la collaboration de plusieurs pays de l'Union européenne. La France y est particulièrement active, mais également le RoyaumeUni où plusieurs organismes de recherche-développement se sont regroupés dans un "forum" agroforestier, dont le réseau expérimental se développe depuis 1987 et totalise actuellement 65 hectares d'agroforesterie pâturée. Les pays méditerranéens (Grèce, Espagne, Portugal) y participent aussi, dans une moindre mesure, ainsi que certains pays tempérés (Pays-Bas, Danemark).

Jusqu'à présent, les recherches ont principalement porté sur les pratiques agroforestières traditionnelles et visaient au développement de techniques respectueuses de l'environnement (en particulier concernant les risques d'érosion ou la surexploitation forestière) et à l'intégration dans le tissu social existant (dans les pays du Sud). Mais de nombreuses questions demeurent en suspens, particulièrement dans le contexte français et européen. Nous les classerons par commodité en :

- questions biotechniques : quels sont les moyens techniques permettant de gérer au mieux une parcelle agroforestière, et quels sont les processus biologiques sous-jacents ? Quels sont les moyens techniques et les processus fondamentaux impliqués, permettant de gérer au mieux un système agroforestier (ou un ensemble de parcelles agroforestières) ?

- questions socio-économiques: comment s'intègre une activité agroforestière dans une exploitation agricole ou dans un aménagement forestier, quels sont les atouts de l'agroforesterie, quelle valeur donner aux bénéfices non-marchands qu'elle induit, quels sont ses impacts sur l'économie publique, quelles réglementations nécessite-t-elle?

- questions écologiques : comment s'intègrent des activités agroforestières dans l'environnement (physique et biologique), peuvent-elles permettre de mieux gérer l'espace rural et le paysage, peuvent-elles participer à une meilleure gestion des risques?

Les situations locales sont très diverses et dépendent souvent plus du contexte socio-économique ou des traditions régionales que de l'environnement physique : bocage breton et haies brise-vent provençales, noyeraies cultivées du Dauphiné et parcours boisés gascons, pare-feux méditerranéens pâturés et pré-bois auvergnats... Ce qui suit aura donc inévitablement un caractère général. Le point commun entre les diverses formes d'agroforesterie traitées ici est la présence, sur une même parcelle, d'arbres et d'autres productions agricoles (cultivées ou pâturées). Nous serons cependant amenés à aborder un aspect débordant cette définition restrictive de l'agroforesterie, celui de la forêt paysanne: la question de l'insertion d'activités agroforestières dans un système d'exploitation agricole rejoint celle, plus générale, des activités forestières des agriculteurs.

Nous développerons principalement les aspects techniques sur lesquels on manque encore de réponses et, nous adressant aux lecteurs de la Revue forestière française, nous privilégierons l'arbre par rapport aux autres constituants du système (herbe ou culture, animal, homme), en renvoyant le lecteur aux deux articles qui suivent. Les idées développées sont issues de divers rapports élaborés pour alimenter la réflexion sur les programmes de recherche en cours d'élaboration (Cailliez et al., 1993 ; Guitton et al., 1993).

\section{LE NIVEAU DE LA PARCELLE}

Dans la parcelle agroforestière se manifestent les interactions entre le peuplement forestier et le peuplement herbacé ou la culture (concurrence pour l'eau et la lumière, cycle des nutriments, décalages phénologiques, effets de bordure...). II s'y pose de nombreuses questions techniques: du fait de la présence de deux strates de végétation, des interventions culturales particulières 
devront être appliquées sur les arbres ou la végétation accompagnatrice; la conduite des troupeaux devra être adaptée. Des itinéraires techniques visant à favoriser chaque élément du système devront être mis au point, contrairement aux pratiques traditionnelles de la monoculture.

\section{Techniques de plantation des arbres}

Du fait de l'histoire des parcelles forestières ou agricoles à convertir à l'agroforesterie, les techniques forestières traditionnelles de plantation ou de régénération sont peu adaptées aux milieux envisagés et à la présence de plantes cultivées ou de bétail. "I se pose différentes questions :

\section{- Caractérisation du milieu}

II y a lieu de mettre au point de nouveaux outils de détermination du milieu et des potentialites, ceux employés classiquement en forêt n'étant pas applicables aux milieux ouverts ou utilisés pour des productions agricoles ou fourragères (Franc, 1993).

\section{- Préparation du terrain avant plantation d'arbres}

Elle doit tenir compte de l'occupation actuelle du sol, ainsi que de son histoire (tassement des horizons profonds, manque de matière organique, rémanence de pesticides et d'herbicides, de fertilisant, présence ou absence de végétaux adventices, de ligneux concurrents, de symbiotes racinaires, d'insectes ou de pathogènes, de petits rongeurs...) (Bailly, 1991; De Champs, 1990).

\section{- Choix des arbres}

Il dépend de l'objectif fixé ; selon qu'il s'agit de production de bois d'œuvre de qualité, de fourrage (feuilles ou fruits), de création d'un microclimat favorable aux autres éléments du système (protection pour les animaux, décalage phénologique de la culture ou de l'herbe) ou de creation d'un paysage, le choix des espèces sera différent. Dans la mesure où l'on s'intéresse a priori à un petit nombre d'arbres, à large espacement, on ne pourra pas effectuer de sélection individuelle par éclaircie, la qualité génétique est donc d'une importance considérable. Les caractéristiques à prendre en compte dans l'amélioration génétique peuvent être différentes de celles recherchées en ambiance forestière : adaptation aux milieux agroforestiers (phénologie, potentiel racinaire), convivialité avec les cultures intercalaires, résistance aux maladies, production, qualité (qualité intrinsèque du bois, angle et grosseur de la branchaison, aptitude à la taille de tormation, ...).

- Contrôle de la végétation concurrente, fertilisation, protection contre les animaux

Ces techniques, très élaborées dans le cas des monocultures, doivent être adaptées aux situations agroforestières. Bien qu'un des objectifs visés par l'agroforesterie concerne souvent une diminution des intrants, la survie du système global (arbres, herbe, animaux) nécessite des interventions plus ou moins importantes selon les situations. Un équilibre est à trouver entre une production satisfaisante et une saine gestion des intrants.

\section{Techniques de conduite des arbres}

La sylviculture classique ayant quasiment exclu toute activité agricole des parçelles forestières, de nouvelles règles de conduite doivent être trouvées, permettant de tenir compte des autres composantes du système.

\section{- Tailles de formation et techniques d'élagage}

Du fait d'une faible densité d'arbres par unité de surface, l'élagage naturel sera fortement réduit. Dans une optique de production de bois de qualité, les meilleures techniques doivent être trouvées (Hubert et Courraud, 1987): date, fréquence, type, intensité des interventions, gestion des blessures et de leurs conséquences. Les rémanents doivent aussi être gérés de manière à tenir compte de la strate basse et, éventuellement, des animaux. 


\section{AUCLAIR - F. CAILLIEZ}

- Techniques d'éclaircie, débroussaillement

Ces techniques seront à déterminer en fonction des objectifs forestiers (bois d'œuvre, bois de feu), pastoraux (survie, engraissement, calendrier fourrager) ou environnementaux (prévention contre les incendies, accueil du public, maintien ou développement de biotopes particuliers) (Étienne et al., 1993).

\section{- Régénération des peuplements}

Les techniques classiques par rejets ou semis sont inadaptées dans les systèmes sylvopastoraux et on doit envisager une conduite des troupeaux particulière (dates et intensité du pâturage), voire une mise en défens temporaire.

Toutes ces techniques doivent être compatibles avec la quantité de travail disponible au sein du système agroforestier, et leur robustesse par rapport à ce facteur doit être appréciée avec soin.

\section{Techniques de conduite de la culture ou de la ressource pastorale}

Ici aussi, de nouvelles règles de conduite de la végétation basse doivent être trouvées, tenant compte de la présence d'arbres sur la parcelle.

- Le choix du type de culture intercalaire devra être déterminé en fonction du cycle d'activité, de manière à rechercher la complémentarité entre strates et à optimiser les interactions. Le choix des espéces intercalaires peut permettre soit un enracinement complémentaire entre arbres et culture, soit un cycle d'activité adapté à la fois aux périodes critiques pour les arbres et la culture. Il y a lieu de rechercher une optimisation des interventions culturales éventuelles (irrigation, désherbage, fertilisation, sursemis) en fonction des différents éléments du système et des objectifs.

- Les interactions entre strates sont extrêmement importantes. Elles peuvent d'une part influencer la quantité produite, par un décalage phénologique dans la croissance de la strate basse en cours d'année, et par une diminution de la production en fin de révolution de la strate arborée. Elles peuvent d'autre part influencer la qualité : qualité de la culture ou composition botanique et valeur nutritive du fourrage dépendent de l'ensoleillement reçu et de l'alimentation hydrique.

- Si les interventions culturales destinées à la strate basse peuvent avoir des effets bénéfiques sur les arbres, qui profitent de l'irrigation, de la fertilisation, ou de la présence de légumineuses, en revanche les travaux mécaniques d'entretien peuvent avoir des effets négatifs sur la texture du sol, ou occasionner des blessures aux racines ou aux troncs.

\section{Techniques de conduite des animaux et du système fourrager}

La conduite des animaux doit tenir compte de l'ensemble des éléments du système, la disponibilité fourragère et la strate arborée. Le calendrier fourrager et la charge instantanée seront à adapter en fonction des objectifs et des conditions de la parcelle.

- La disponibilité de la ressource pastorale est influencée par la présence d'arbres, et la conduite du troupeau doit tenir compte du décalage phénologique : la perte de croissance de la ressource en début de saison a lieu à une période où l'éleveur peut généralement trouver une ressource importante sur d'autres parcelles; par contre, le prolongement de croissance en période estivale peut être mis à profit, en particulier en région méditerranéenne où la ressource fourragère estivale disponible par ailleurs est généralement très limitée.

- La qualité de la ressource est également influencée par les arbres. D'une part, la composition botanique est influencée par l'ombrage ou la compétition racinaire. Mais d'autre part, dans la gestion des ressources boisées difficiles à valoriser (Midi méditerranéen), le pâturage peut avoir pour objectif l'entretien de la végétation arbustive, dont la qualité nutritive est généralement très réduite (Meuret, 1989). Une conduite particulière des troupeaux est alors nécessaire, conciliant les 
impératifs de l'éleveur (croissance ou entretien des animaux) avec la faible qualité nutritive de la ressource. Une ressource fourragère complémentaire peut s'avérer nécessaire, soit par sursemis et/ou fertilisation sur la parcelle, soit par apport extérieur.

- La conduite des animaux doit aussi être adaptée à la présence d'arbres. Les protections individuelles ont une efficacité variable et une mise en défens peut s'avérer nécessaire à certaines périodes critiques de la vie des arbres, ou du troupeau. La charge moyenne et la charge instantanée doivent être adaptées à la fois aux caractéristiques des animaux (espèce, race, âge...), à la quantité de fourrage disponible, et aux arbres (espèces, âge).

Si certaines des questions pratiques évoquées ci-dessus peuvent être résolues par la connaissance des systèmes traditionnels, quelques sujets de recherche prioritaires concernant l'agroforesterie peuvent être dégagés.

Depuis une dizaine d'années, les organismes de recherche français ont installé plusieurs réseaux de dispositifs expérimentaux agroforestiers :

- en matière d'agroligniculture, le CEMAGREF a installé environ 100 hectares de plantations expérimentales avec pâturage en Auvergne et au Pays Basque, I'INRA 22 hectares d'agroligniculture pâturée en Languedoc-Roussillon et en Midi-Pyrénées, ainsi que 6 hectares de plantations d'arbres avec cultures intercalaires (Guitton et al., 1990 ; Guitton et al., 1993) ;

- I'INRA suit un réseau de 122 placettes totalisant près de 1500 hectares d'expérimentations sylvopastorales en région méditerranéenne, et le CEMAGREF trois essais d'ouverture de plantations résineuses au pâturage sur 25 hectares en Auvergne ;

- à ces grandes expérimentations s'ajoutent des dispositifs expérimentaux plus fins visant à répondre à certaines questions scientifiques ou techniques particulières, installés par l'AFOCEL, le CEMAGREF, I'IDF, I'INRA.

Cependant, les organismes de recherche ne peuvent pas multiplier les expériences en vraie grandeur, bien qu'elles soient nécessaires, ne serait-ce que pour communiquer avec les praticiens ; ceci est plutôt le rôle des organismes de développement. Au niveau de la parcelle, la Recherche doit particulièrement s'attacher aux mécanismes mis en jeu dans les interactions entre éléments du système. Elle doit également fournir des modèles de fonctionnement : modèles de croissance ou modélisation globale des parcelles agroforestières, voire des systèmes agroforestiers. Un des objectifs essentiels des recherches à caractère biotechnique décrites ici est d'alimenter par des données quantitatives et qualitatives fiables les études socio-économiques. Sans ces données biologiques concrètes, les modèles économiques n'auraient aucune possibilité de fonctionner et d'aboutir à des débouchés pratiques.

\section{Produire du bois de qualité avec des arbres forestiers en "croissance libre"}

Très peu d'études ont été menées sur la production des arbres forestiers dans des situations proches de la "croissance libre", c'est-à-dire sans concurrence entre eux. Dans les systèmes agroforestiers traditionnels, comme par exemple les herbages plantés de pommiers en Normandie ou les noyeraies du Dauphiné, l'objectif de production de la strate arborée concerne généralement d'autres produits que le bois (fruits ou fourrage). Si l'on envisage des systèmes producteurs de bois de qualité, de nombreuses questions se posent:

- La sélection génétique: les programmes d'amélioration des arbres forestiers de I'INRA et de l'AFOCEL orientent leurs recherches essentiellement vers la production de bois de qualité :

- Les principales espèces pouvant intéresser l'agroforesterie sont les suivantes: Chêne rouge, Merisier, Mélèzes, Noyers et, dans une moindre mesure, Pin laricio, Douglas, Cèdres, Cyprès qui font l'objet d'un programme d'amélioration, et pour lesquels certains des dispositifs expérimentaux peuvent dès à présent intéresser l'agroforesterie, étant installés en milieu agricole dans des situations de concurrence faible. 


\section{AUCLAIR - F. CAILLIEZ}

- Un effort particulier va porter sur des espèces non encore prises en compte dans ces programmes et en particulier les Frênes.

- On peut envisager de s'intéresser également à l'avenir d'autres espèces telles que les Érables et les fruitiers forestiers.

- La mise au point de modèles prédictifs de la croissance du tronc, prenant en compte la station (et son hétérogénéité), la densité (la notion de "hauteur dominante" disparaît lorsque l'on descend en dessous de 100/ha), les effets d'échelle, de bordure ou de lisière, les interactions avec la végétation accompagnatrice.

- L'intégration dans ces modèles des aspects concernant la qualitè du bois, influencée par la largeur, l'irrégularité, et la dissymétrie des accroissements, par les élagages et tailles de formation (effets sur les profils microdensitométriques et sur le dessin et la coloration du bois).

- La mise au point de modèles prédictifs de la croissance du houppier : le développement architectural aérien sera plus vigoureux qu'en forêt, du fait d'une fertilité parfois supérieure et de l'absence de compétition entre arbres, et sera modifié par les opérations de taille et éventuellement par la présence de tubes de protection. Ce développement du houppier intéresse à la fois les utilisateurs du bois - par son influence sur la quantité produite par le système photosynthétique ainsi que sur la qualité par l'intermédiaire des tailles de formation - et les gestionnaires de la végétation basse - par son influence, directe (par compétition) ou indirecte (sur le microclimat), sur la quantité et la qualité de la strate basse.

- L'étude de la croissance du système souterrain, particulièrement important dans les interactions entre arbres et végétation basse : les connaissances sont actuellement extrêmement lacunaires, en particulier sur les terrains considérés (fertilité, présence de semelle de labour, absence de systèmes ectomycorhiziens...).

- L'influence des tubes-abris, utilisés pour protéger les arbres contre le bétail : ces tubes ont un effet sur la croissance juvénile des arbres ("effet de serre"), qui peut être modulé par de nouvelles conceptions de tubes. Cette phase juvénile étant particulièrement importante pour la mise en place du système, des études écophysiologiques fines des arbres en tubes sont encore nécessaires. Le comportement des arbres à la sortie des tubes, et pendant les années qui suivent, est particulierement important et encore insuffisamment connu (Dupraz et al., 1993).

Grâce aux collaborations entre organismes de recherche, la France développe actuellement des modèles originaux intégrant lois de croissance, architecture, qualité du bois (Bouchon, 1994). Ces types de modèles, s'intéressant aux arbres individuels, sont particulièrement adaptés aux conditions de l'agroforesterie, et leur extension à des niveaux plus globaux (parcelle, paysage) est en cours d'étude.

\section{Maîtrise des associations arbre-herbe et des interactions entre strates de végétation}

L'originalité des systèmes agroforestiers est la présence en un même lieu d'arbres et d'autres végétaux. Les interactions, en particulier leurs aspects écophysiologiques, doivent être étudiées en détail pour une meilleure gestion des associations.

\section{- Compétition pour la lumière}

L'effet le plus immédiatement visible des arbres sur la végétation basse est l'effet d'ombrage. La quantité et la qualité de la lumière seront modifiées après la traversée des houppiers, avec de multiples conséquences sur le microclimat régnant sous les arbres, et donc sur la croissance, la composition botanique et la qualité de la végétation basse. Les modifications de température ont également des effets sur la respiration, la transpiration et la phénologie (Baldy et al., 1993).

\section{- Compétition pour l'eau}

Dans la plupart des milieux (y compris les "montagnes humides" comme par exemple le Massif Central), l'eau est un facteur limitant la production, et sa gestion est souvent problématique. La 
végétation basse a des effets divers sur la croissance des arbres (en fonction du milieu ou de la saison), et inversement, les arbres ont un effet sur l'interception des pluies, l'évaporation, l'écoulement le long des troncs. La répartition de l'énergie incidente crée une demande climatique différente selon la strate et au cours du temps. La physiologie et le comportement hydrique de chaque élément du système sont soit concurrentiels, soit complémentaires selon les espèces, et le choix des cultures associées est particulièrement important. Ici encore, la connaissance de la structure et du fonctionnement des systèmes racinaires des deux strates est primordiale.

\section{- Fonctionnement minéral}

La compétition pour les éléments minéraux est un complément au point précédent. La nature du sol et son histoire agricole ou forestière justifient parfois une fertilisation dans l'optique d'une production agricole ou animale, induisant un effet bénéfique sur la croissance des arbres. $A$ contrario, l'optique d'extensification vise une réduction des intrants, menant à une compétition plus importante entre éléments du système. Les cycles bio-géochimiques sont modifiés par la présence des arbres : par exemple, l'absorption des nitrates en profondeur, l'apport de litière des feuilles, la fixation symbiotique des légumineuses cultivées à l'ombre contribuent à modifier le cycle de l'azote. Le phosphore est lui aussi très important dans la compétition entre strates. Des recherches sont nécessaires pour avoir une bonne connaissance des mécanismes et pour quantifier leurs effets.

L'ensemble de ces phénoménes devrait influer la gestion des intrants. L'utilisation raisonnée des produits chimiques exige de bien comprendre non seulement le fonctionnement de chaque espèce séparément, mais également leurs interactions.

\section{Maîtrise des interactions entre arbres et animaux}

Une des réticences les plus importantes de la sylviculture traditionnelle vis-à-vis du pâturage concerne les dégâts (écorçage, abroutissement, piétinement). Ceux-ci sont relativement bien maîtrisés par des solutions techniques, certes encore à améliorer, telles que les tubes-abris avec ou sans tuteur complémentaire ou garde de fil barbelé. L'efficacité des protections est aussi très liée à la conduite des troupeaux et des études de comportement animal doivent être poursuivies. Une bonne conduite des animaux a des effets bénéfiques tels que le contrôle par ingestion ou piétinement de la végétation concurrente en forêt. Dans l'étude des cycles bio-géochimiques, la participation des déjections animales peut être particulièrement importante.

\section{LE SYSTÈME AGROFORESTIER}

Nous définirons ici schématiquement un "système agroforestier" comme un ensemble de parcelles agroforestières gérées conjointement au sein d'une même "unité économique". Cette définition est cependant très restrictive et il est particulièrement difficile de donner une définition unique du système agroforestier. En effet, il existe de nombreuses formes différentes, comme par exemple:

- Une exploitation agricole comportant certaines parcelles agroforestières, mais éventuellement aussi des parcelles forestières pures ("forêt paysanne"). L'introduction d'une production forestière dans une exploitation agricole génère une diversification des produits mais introduit également un besoin de réajustement dans le système de production et modifie substantiellement le pas de temps de programmation de l'exploitation.

- Un système d'élevage comportant certaines parcelles agroforestières et, éventuellement, des parcelles forestières pures. L'association de la forêt et du pâturage crée des ressources nouvelles et, en particulier, des décalages phénologiques à des moments clefs du calendrier fourrager. Notons que la frontière entre parcelles forestières et agroforestières est relativement 
floue: de nombreux éleveurs introduisent une partie de leur troupeau dans les parcelles boisées à certaines périodes de l'année ou dans certaines conditions particulières.

- Une "unité de gestion forestière" (massif forestier domanial, forêt communale, forêt privée...) intégrant des activités d'élevage sur une ou plusieurs parcelles. L'ouverture de la forêt à une activité pastorale rend nécessaire la prise en compte de critères non traditionnels dans l'aménagement forestier. Un compromis entre la production de bois (ou de services) et la production complémentaire liée à l'activité agricole doit être trouvé.

- Plus généralement, un "système de gestion multi-usages". L'agroforesterie, étant toujours liée à une diversification des produits, entraîne souvent une multiplication des usages potentiels. Ceci pose la question de l'organisation temporelle et spatiale de ces activités dans un but de limitation des conflits et de complémentarité des tâches.

Dans de nombreux cas, les limites géographiques d'un "système agroforestier" sont floues ou variables au cours du temps. En effet, il s'agit généralement d'une organisation complexe dans laquelle des groupes sociaux différents font intervenir des itinéraires techniques variés sur un espace variant lui aussi dans le temps.

D'une part, on s'adresse généralement à un grand nombre d'acteurs. S'il existe des cas relativement simples comme par exemple une exploitation agricole qui diversifie ses activités par la production de bois, en revanche dans de nombreuses situations plusieurs acteurs différents vont être impliqués: propriétaire foncier (privé ou public), propriétaire du troupeau, exploitant agricole, berger, sylviculteur. À ceux-ci s'ajoutent chasseurs, apiculteurs, promeneurs, représentants de la collectivité s'intéressant à la protection contre les incendies, à la qualité des eaux, au paysage, à la biodiversité... Le nombre et les caractéristiques des acteurs varient parfois au cours du temps.

D'autre part, la gestion de l'espace devient complexe. Si l'allocation de parcelles forestières s'effectue de manière relativement "simple" grâce à un aménagement forestier ou un plan simple de gestion, la superposition des usages va compliquer le système. Certains acteurs utiliseront une parcelle à une période donnée, en fonction des conditions écologiques mais aussi en fonction des conditions climatiques une année donnée. Le calendrier fourrager peut par exemple être complètement modifié une annèe du fait d'une pluviométrie abondante ou au contraire d'une sécheresse, et l'éleveur sera amené à rechercher une ressource pastorale à l'extérieur du système (par transhumance, ou apport de fourrage). Dans d'autres cas, la superposition d'usages ne se fera qu'à une certaine période bien précise, les acteurs ayant leur activité principale à l'extérieur du système (cas de la transhumance inverse).

Un troisième point important concerne les échelles de temps, chacun des acteurs raisonnant à une échelle différente. Un calendrier fourrager se raisonne à une échelle inférieure à la décade et varie d'une année à l'autre du fait des conditions climatiques. Une exploitation agricole raisonne à l'échelle de la saison ou de l'année, mais la vie des arbres fruitiers est de l'ordre de la décennie, et celle des arbres forestiers de plusieurs décennies, vaire de plusieurs siècles. Toutes les activités concernées, pression touristique, activités de chasse, apiculture, gestion des risques d'incendie, ont également leur calendrier propre.

L'ensemble de ces considérations souligne l'importance des recherches pluridisciplinaires, impliquant non seulement biologistes, agronomes et forestiers, mais aussi sociologues, économistes, écologistes. Il s'agit de définir des règles de gestion multi-usages et multi-usagers, et de s'intéresser à un réel "aménagement agroforestier", complexe, qui ne soit pas une simple juxtaposition d'un système d'exploitation agricole adapté à la présence d'arbres, d'un système de gestion pastorale adapté à la présence d'arbres et d'un aménagement forestier adapté à la présence d'animaux. La question cruciale qui se pose concerne l'affectation des parcelles dans l'espace et dans le temps, et des travaux à y conduire, en tenant compte de l'ensemble des abjectifs. 
À cela s'ajoutent d'autres questions :

- celle de la mécanisation : l'agriculteur dispose-t-il des machines nécessaires pour le travail sur les arbres forestiers, peut-il les modifier à peu de frais, comment calculer l'amortissement, doitil faire appel à une entreprise extérieure? Le sylviculteur dispose-t-il des moyens nécessaires pour effectuer des débroussaillages, des sursemis ou une fertilisation pour faciliter le passage du bétail sur les parcelles?

- celle de la pluriactivité : l'agriculteur peut-il diversifier ses activités vers la gestion forestière non seulement des parcelles lui appartenant, mais également à l'extérieur de son exploitation ? Le sylviculteur, habitué à gérer des travaux forestiers, peut-il intégrer dans sa gestion la participation d'animaux (et de bergers) à l'entretien des pare-feux plutôt que celle d'une équipe de forestierssapeurs?

- celle de la gestion du temps de travail: les techniques de conduite des arbres (élagage, tailles) sont-elles compatibles avec les autres activités de l'agriculteur?

Ces questions renvoient à celles relatives à la rentabilité des activités forestières des agriculteurs, à la place des bois dans les exploitations agricoles, à l'insertion des activités forestières dans l'économie des ménages agricoles, qui sont traitées dans l'article suivant (p. 152).

\section{VERS UNE GESTION INTÉGRÉE DE L'ESPACE RURAL}

Sans avoir la prétention de vouloir couvrir toutes les questions d'ordre écologique, il est important de souligner ici quelques aspects concernant la gestion spatio-temporelle des espaces à usages multiples.

Si des reboisements à grande échelle ont été par le passé accusés de contribuer à la désertification de certaines vallées de basse ou moyenne montagne et si la prolifération de boisements "en timbres-poste" a été largement critiquée, il est indispensable d'intégrer les questions concernant l'écologie du paysage dans la problématique générale de l'agroforesterie. Comment les activités agroforestières s'intègrent-elles dans le paysage et comment peuvent-elles contribuer à la gestion de l'environnement?

Bien que les systèmes agroforestiers soient souvent décrits comme des modes de gestion respectueux de l'environnement, plus extensifs que les systèmes traditionnels, ne doivent-ils pas être au contraire considerés comme des systèmes plus intensifs, puisqu'ils impliquent une exploitation maximale du système, par utilisation de l'ensemble des strates de végétation, et par une prospection racinaire de l'ensemble des horizons utilisables?

L'agroforesterie est souvent décrite comme un système permettant une réduction des intrants, mais, dans les lignes qui précèdent, nous avons évoqué à plusieurs reprises la possibilité, voire la nécessité, d'intervenir par divers travaux de débroussaillement ou de fertilisation. Le rôle du bocage et des brise-vent sur le cycle de l'eau et sur les dégâts dus à l'érosion torrentielle ou éolienne a été bien étudié, en particulier à l'occasion des remembrements bretons ou dans les vergers provençaux. Le rôle des massifs forestiers sur les cycles bio-géochimiques commence aussi à être mieux compris. Il est par exemple probable que le prélèvement de nitrates par les arbres à large espacement soit relativement peu efficace et que des pertes par drainage soient inévitables; le rôle des cultures intercalaires est sans doute plus déterminant et, dans les cas de pâturage, le comportement devient voisin de celui d'une prairie. Cependant, on manque encore de références sur un éventuel rôle de "dépollution" que peuvent jouer les arbres en situation agroforestière: sur le cycle de l'eau et sa qualité, sur le ruissellement et la migration vers les nappes... (Ranger, in Cailliez et al., 1993).

La biodiversité est devenue une préoccupation importante pour les gestionnaires de l'espace rural et de la forêt (DERF, 1993). Afin de conserver la diversité biologique en matière de faune et de flore, 


\section{AUCLAIR - F. CAILLIEZ}

une hétérogénéité des structures est préconisée, les zones de discontinuité telles que les lisières, clairières, limites de peuplement étant des milieux de richesse écologique particulièrement grande. II reste cependant à trouver un équilibre entre une mosaïque excessive, source de difficultés de gestion, et une trop grande homogénéité.

II ne faut cependant pas négliger d'éventuels effets pervers de l'augmentation de la diversité faunistique. Si l'on s'interroge par exemple sur la dynamique des populations d'arthropodes nuisibles aux cultures, les systèmes agroforestiers peuvent, selon la nature de la culture et selon la composition spécifique du système, fonctionner tantôt comme réservoir d'auxiliaires, c'est-à-dire d'arthropodes entomophages (parasites ou prédateurs) susceptibles de limiter les pullulations de certains des ravageurs de la culture, tantôt au contraire fonctionner comme des réservoirs d'arthropodes phytophages potentiellement nuisibles à cette dernière, tantôt enfin jouer ces deux rôles simultanément (Du Merle in Cailliez et al., 1993). On peut ainsi créer avec un système agroforestier une nouvelle dynamique non seulement des insectes, mais aussi des champignons ou des maladies, et un accroissement de l'avifaune ou du gibier peut entraîner des dégâts aux arbres ou aux cultures.

En plus des différents facteurs de risque décrits ci-dessus, en région méditerranéenne, nous devons prendre en compte le risque d'incendie. L'abandon de toute activité agricole augmente généralement ce risque et le maintien d'une population rurale est un des éléments qui permettent sa réduction. De nombreuses expériences montrent qu'on peut obtenir un excellent contrôle de la végétation basse (qui est un des principaux facteurs d'aggravation du risque) à un moindre coût grâce à une gestion sylvopastorale appropriée. Cependant, cette gestion est souvent difficile à mettre en œuvre et les calculs économiques délicats, en particulier concernant la rémunération des surplus (si le débroussaillement est mieux fait, et plus durable, comment estimer sa valeur ?) ou plus généralement l'estimation de la valeur des biens non marchands, ou celle de l'efficacité (les risques sont-ils réellement diminués, et quelle valeur attribuer à cette diminution des risques ?).

\section{CONCLUSIONS}

Dans le contexte socio-économique actuel, les recherches françaises sur l'agroforesterie prennent plus d'ampleur; mentionnons par exemple:

- Un programme de recherche intitulé "utilisation alternative des terres agricoles avec des arbres à croissance rapide", qui a demarré en 1993 avec une aide financière de la Commission européenne, regroupant 18 instituts de recherche-développement de 6 pays européens. La France y participe pour une grande part, avec comme acteurs principaux I'INRA (coordonnateur du projet), le CEMAGREF, I'IDF, I'AFOCEL, et le CIRAD (Auclair, 1994).

- Un programme de recherche intégré sur la forêt paysanne, l'agroligniculture et le sylvopastoralisme, développé actuellement par l'INRA en collaboration avec le CEMAGREF (Cailliez et al., 1993).

Nous avons fait apparaître ici principalement des questions d'ordre technique ; cependant, dès que l'on se place à un niveau supérieur à celui de la parcelle, au niveau du système agroforestier ou à celui de la région, on aborde des questions intimement liées au contexte socio-économique. La réforme de la politique agricole commune de 1992 et les accords commerciaux internationaux (GATT), mais aussi les accords de Rio sur l'environnement, ont une influence certaine sur les questions qui se posent actuellement.

Si les discours des pouvoirs publics, des élus, des organismes professionnels incitent à une plus grande intégration des activités agricoles et forestières, il se pose de nombreuses questions d'ordre économique, relatives à la production ligneuse mais aussi à la production de biens non 


\section{L'avenir de l'agroforesterie en France}

marchands, ainsi que d'ordre juridique (statut des acteurs de ces systèmes complexes), contractuel (gérant les relations entre acteurs), réglementaire, fiscal (impact des mesures nationales ou communautaires), ou foncier... Ces questions sont développées dans les articles qui suivent.
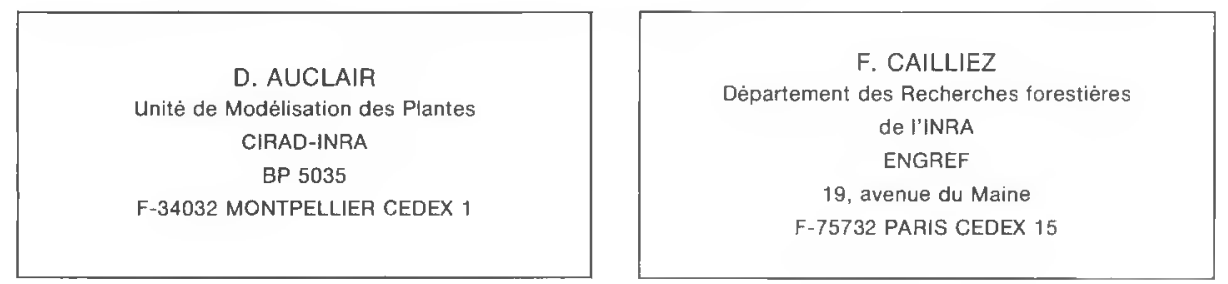

\section{BIBLIOGRAPHIE}

AUCLAIR (D.). - Issues in modelling forestry and agricultural systems. In : Land use science - its role in the development of decision support systems in rural areas. Aberdeen, 29-30/03/1993. - pp. 27-33.

AUCLAIR (D.) Ed. - Utilisation alternative des terres agricoles avec des arbres à croissance rapide. Rapport d'avancement des travaux 1993. - INRA - CCE, 1994. - $386 \mathrm{p}$.

BAILLY (A.). - Le Reboisement en plaine méditerranéenne. - Informations-Forét, fasc. 420, 1991, pp. $237-251$.

BALDY (C.), DUPRAZ (C.), SCHILIZZI (S.). - Vers de nouvelles agroforesteries en climats tempérés et méditerranéens. 1 - Aspects agronomiques. - Cahiers Agricultures, n ${ }^{\circ} 2,1993$, pp. 375-386.

BIDEAU (E.), GONZALEZ (G.), ANGIBAULT (J.-M.). — Les Dégâts sylvicoles des cervidés: déterminisme, protection. - Rapport INRA - IRGM, 1993. - 9 p.

BOUCHON (J.) Ed. - Lois de croissance et architecture. Compte-rendu d'AIP. — Les Colloques de I'INRA, 1994.

CAILLIEZ (F.), CAVAILHES (J.), HUBERT (B.), MONTARD (F.-X. de). - Forêt paysanne, agro-ligniculture et sylvopastoralisme. Rapports d'étape avril et novembre 1993. - Paris : INRA, 1993.

CHAMPS ( $\mathrm{J}$. de). - Les Boisements en résineux des terres délaissées par l'agriculture. - Informations-Forêt, $n^{\circ} 4$, fasc. 398, 1990, pp. 285-303.

DIRECTION DE L'ESPACE RURAL ET DE LA FORÊT. - Définition d'une politique nationale de prise en compte de la biodiversité dans la gestion forestière. - Circulaire DERF/SDEF/n ${ }^{\circ} 3002,1993 .-19 \mathrm{p}$.

DUPRAZ (C.), AUCLAIR (D.), GUITTON (J.-L.). - Vingt ans de recherche agroforestière en Nouvelle-Zélande : quels enseignements pour l'Europe? $1^{\text {re }}$ partie: L'agroforesterie néo-zélandaise. - Revue forestière française, vol. XLIV, $n^{\circ} 6,1992$, pp. 523-538.

DUPRAZ (C.), GUITTON (J.-L.), RAPEY (H.), BERGEZ (J.-E.), MONTARD (F.-X. de). - Broad-leaved tree plantation on pastures: the treeshelter issue. In: Proceedings of the 4 th international symposium on Windbreaks and Agroforestry, Viborg (DK), 1993. - pp. 106-111.

ÉTIENNE (E.), ARMAND (D.), JULLIAN (P.), NAPOLEONE (M.). - Un contrat d'entretien de pare-feu par des moutons; bilan 1987-1992. - Rapport INRA, 1993. - $48 \mathrm{p}$.

FRANC (A.). - Site evaluation and species choice in aftorestation. In: Silvicultural implications for the establishment and early maintenance of new woodlands. Edinburgh, 13-14/10/1993. — 8 p.

GOLD (M.A.), HANOVER (J.W.). - Agroforestry systems for the temperate zone. - Agroforestry systems, $n^{\circ} 5$, 1987, pp. 109-121.

GUITTON (J.-L.), BRETIÉRE (G.), SAAR (S.). - Culture d'arbres à bois précieux en prairies pâturèes en moyenne montagne humide. - CEMAGREF, 1990. - 117 p. (Études Forêt $\mathrm{n}^{\circ} 4$ ).

GUITTON (J.-L.), DUPRAZ (C.), MONTARD (F.-X. de), RAPEY (H.), - Vingt ans de recherche agroforestière en Nouvelle-Zèlande: quels enseignements pour l'Europe? $2^{2}$ partie : Les pratiques agroforestières néo-zélandaises sont-elles transposables en France? - Revue forestière française, vol. XLV, $\mathrm{n}^{\circ} 1,1993, \mathrm{pp} .43-58$.

HUBERT (M.), COURRAUD (R.). - Élagage et tailles de formation des arbres forestiers. - Paris : Institut pour le Développement forestier, $1987 .-292 \mathrm{p}$.

JARVIS (P.G.) Ed. - Agroforestry - principles and practice. - London : Elsevier, 1991 - 356 p.

KNOWLES (R.L.). - New Zealand experience with silvopastoral systems : a review. In : Agroforestry - principles and practice / P.G. Jarvis Ed. . - London : Elsevier 1991. - pp. 251-267.

McDICKEN (K.G.), VERGARA (N.T.) Eds. - Agroforestry classification and management. - New York : J. Wiley, 1990. $-382 \mathrm{p}$.

MEURET (M.). - Feuillages, tromages et flux ingèrès. — INRA; Faculté des sciences agronomiques de Gembloux, 1989. - $229 \mathrm{p}$. (Thèse doctorat).

ZHU (Z.), CAII (M.), WANG (S.), JIANG (Y.), Eds. - Agroforestry systems in China. - Chinese academy of Forestry ; International Canada development research center, 1991. $-216 \mathrm{p}$. 